\title{
Right and Left Medial Orbitofrontal Volumes Show an Opposite Relationship to Agreeableness in FTD
}

\author{
Katherine P. Rankin ${ }^{a}$ Howard J. Rosen ${ }^{a}$ Joel H. Kramer ${ }^{a}$ \\ Guido F. Schauer ${ }^{a}$ Michael W. Weiner ${ }^{b}$ Norbert Schuffb Bruce L. Miller ${ }^{a}$ \\ aUniversity of California San Francisco, bSan Francisco VA Magnetic Resonance Spectroscopy Unit, \\ San Francisco, Calif., USA
}

\section{Key Words}

Frontotemporal dementia - Orbitofrontal cortex ·

Agreeableness $\cdot$ Social behavior $\cdot$ Personality

\begin{abstract}
Recent investigations of the neuroanatomy of complex social behaviors suggest that the underlying brain circuits involve multiple cortical and subcortical structures. The neuroanatomic origins of agreeableness have not yet been clearly elucidated. However, frontotemporal dementia (FTD) patients can evidence dramatic alterations in agreeableness arising from frontal and temporal lobe damage. Based on previous research, we hypothesized that agreeableness would be negatively correlated with left medial orbitofrontal cortex size and positively correlated with right amygdala volume. First-degree relatives of 27 FTD patients (diagnosed according to the Lund-Manchester criteria) were asked to fill out the NEOFive Factor Inventory to assess the patients' current level of agreeableness, a construct comprised of the facets trust, straightforwardness, altruism, compliance, modesty, and tender-mindedness. These patients underwent $\mathrm{T}_{1}$-weighted $\mathrm{MRI}$ imaging, and gray matter volumes for right and left orbitofrontal lobes and amygdalas were
\end{abstract}

\section{KARGER}

Fax +41613061234

E-Mail karger@karger.ch

www. karger.com
(C) 2004 S. Karger AG, Basel

Accessible online at: www. karger.com/dem derived via segmentation and region of interest tracing, normalizing for total intracranial volume. Regression analysis revealed that $38 \%$ of the variance in the NEO agreeableness score was predicted by a model in which right orbitofrontal volume ( $\beta=0.731$ ) was positively correlated with agreeableness, and left orbitofrontal lobe volume $(\beta=-0.638)$ was negatively correlated with agreeableness $(p<0.01)$. Contrary to our hypothesis, amygdala volume did not significantly predict agreeableness. This finding partly replicates a previous study that used a different measure of social functioning, the Interpersonal Adjective Scale, to delineate a left frontalright amygdala circuit for agreeableness. These data support the hypothesis that regulation of agreeableness arises from a balanced, mutually inhibitory circuit involving both hemispheres.

Copyright $@ 2004$ S. Karger AG, Basel

\section{Introduction}

Frontotemporal dementia (FTD) patients can evidence dramatic alterations in interpersonal warmth and agreeableness arising from frontal and temporal lobe damage [1-3]. Agreeableness, or the ability to maintain
Katherine P. Rankin, $\mathrm{PhD}$

UCSF Memory and Aging Center

350 Parnassus Avenue, Suite 706

San Francisco, CA 94143-1207 (USA)

Tel. +1 415476 8592, Fax +1 415476 4800, E-Mail krankin@memory.ucsf.edu 
positive social relationships via cooperation and attention to social norms, is a complex but adaptive social behavior that is likely to be mediated by brain circuits involving multiple cortical and subcortical structures. Though the neuroanatomic substrates of agreeableness have not been directly studied, one FTD case study suggested that damage to temporal lobe structures, particularly in the nondominant hemisphere, may result in the loss of empathy and warmth [4]. A voxel-based morphometry analysis on FTD patients revealed that atrophy in bilateral temporal lobe and medial orbitofrontal structures correlated with loss of cognitive empathy, and that atrophy to the temporal pole correlated significantly with loss of emotional empathy [5].

In a pilot study using a personality questionnaire (the Interpersonal Adjective Scales) [6], Rankin et al. [7] previously showed that interpersonal warmth was negatively correlated with left frontal volume and positively correlated with right amygdala volume in FTD. We desired to investigate whether this same relationship would appear with an alternative instrument (the NEO-Five Factor Inventory (NEO-FFI)) [8], measuring a similar behavioral construct, i.e. agreeableness. Based on the results of Rankin et al. [7], we hypothesized that agreeableness would be negatively correlated with left orbitofrontal cortex (OFC) volume and positively correlated with right amygdala volume. Specifically, we analyzed the relationship between NEO agreeableness and brain volume in both orbitofrontal lobes and amygdalae to clarify the function of each structure in the circuit according to its hemispheric laterality.

\section{Methods}

\section{Subjects}

Twenty-seven patients were recruited through the Memory and Aging Center at the University of California San Francisco because they had been diagnosed with FTD according to the Lund-Manchester criteria [9] (age: $64.9 \pm 9.8 ; 19$ males $/ 8$ females). This group included both patients with predominantly temporal or frontal damage, but none with the left frontal variant (or "primary progressive aphasia') [10]. Extensive behavioral, neuropsychological, and neuroimaging diagnostic evaluations were performed by a multidisciplinary team.

\section{Procedures}

Patients were identified from the clinic subject pool by diagnosis and recruited as potential study participants. The subjects and caregivers signed an institutional review board-approved research consent form including an agreement to fill out questionnaires for research purposes.

The NEO Personality Inventory is a psychometrically validated personality assessment tool that measures 5 personality constructs (neuroticism, extraversion, openness, agreeableness, and conscien-

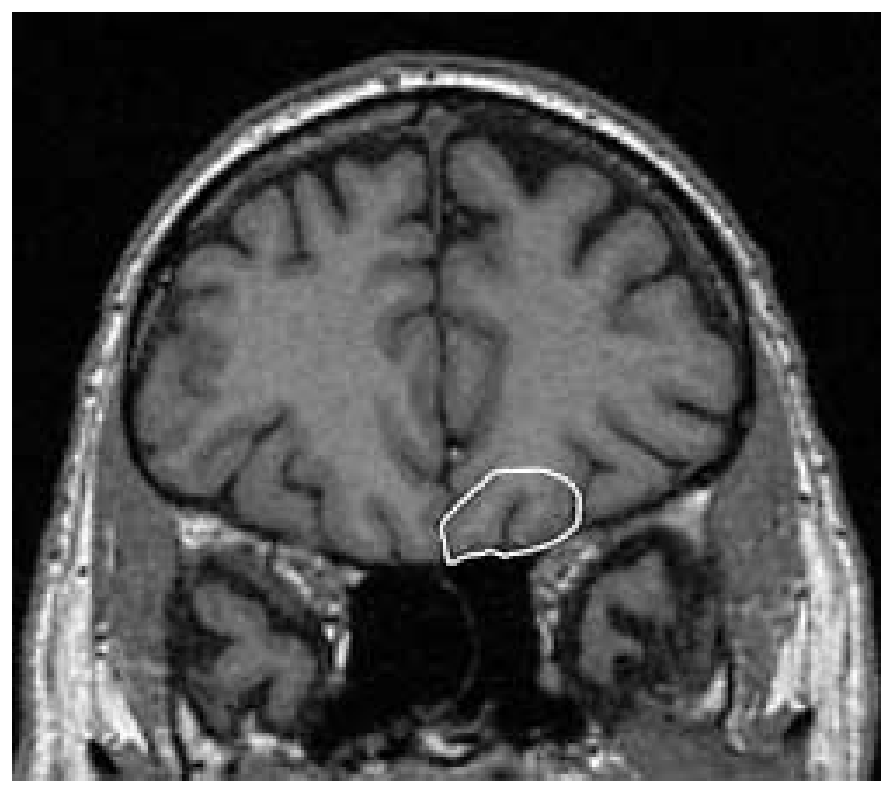

Fig. 1. Boundaries of manual OFC ROI marking, coronal view (figure represents simulated marking, rather than actual ROI marks).

tiousness) $[8,11-13]$; it has been demonstrated to effectively characterize personality in both normal and clinical populations. The 5 factors of the NEO Personality Inventory may be interpreted independently, enabling the assessment of the construct of agreeableness. First-degree relatives were asked to fill out the NEO-FFI to assess the patients' current level of agreeableness. Raters were selected on a case-by-case basis; spouses were used whenever possible (73\%), an adult son or daughter (24\%), and in one case, a sibling caregiver.

These patients underwent $T_{1}$-weighted structural MRI imaging and their MP-RAW images were segmented into gray matter, white matter, and cerebrospinal fluid (CSF). Regions of interest (ROIs) were hand-traced on these images to determine gray matter volumes. Bilateral ROIs included medial orbitofrontal lobes (fig. 1) and the amygdalae. The posterior boundary for the OFC was the first slice anterior to the optic chiasm, and the anterior boundary was the last slice on which the temporal lobe could be seen on either hemisphere. The superior boundary for the OFC was the superior rostral sulcus, on the medial frontal surface, and the lateral boundary was the first orbital sulcus lateral to the olfactory sulcus. Inferiorally and medially, CSF borders the OFC. A binary image was created for the OFC as outlined in the coronal plane, and it was superimposed on the segmented tissue image for that subject to obtain a gray matter volume for the structure. This was not necessary to do for the amygdala, which is all gray matter. The anterior boundary of the amygdala was defined by the closure of the sylvian fissure (endorhinal sulcus). The medial and superior boundaries were defined by CSF medial to the temporal lobe with the extra requirement that no tissue be included superior to the endorhinal sulcus. The lateral boundary was the graywhite border of the amygdala. This approach is essentially the same as previous methods used to obtain amygdala volumes [14]. Corrections were made for differences in head size by normalizing the regional volume using the total intracranial volume, which is the 

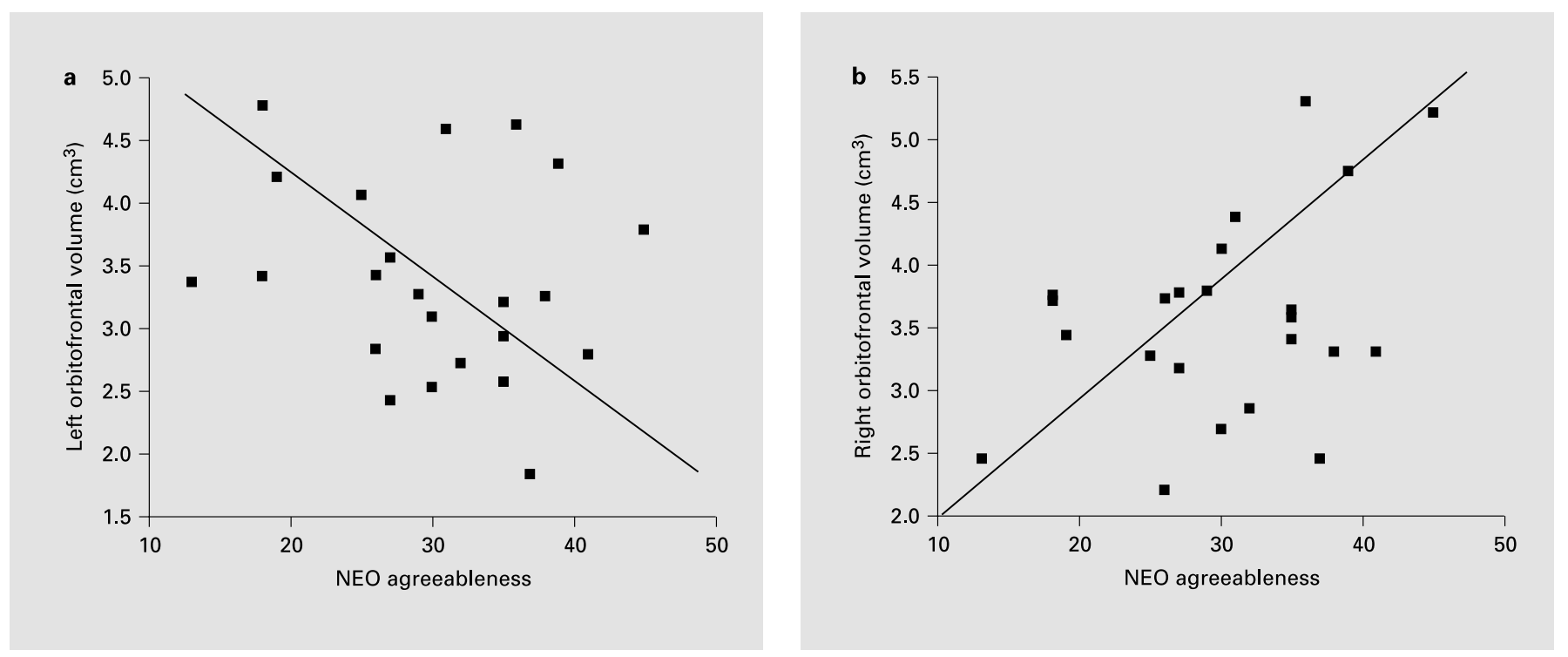

Fig. 2. a Plot of left orbitofrontal volume against the NEO agreeableness score, with regression line $\left(\mathrm{R}^{2}=0.38\right.$, $\beta=-0.638, p<0.01)$. b Plot of right orbitofrontal volume against the NEO agreeableness score, with regression line $\left(\mathrm{R}^{2}=0.38, \beta=0.731, \mathrm{p}<0.01\right)$.

sum of all tissue and fluid volumes measured inside the skull (obtained from the segmented image).

A linear regression analysis was run entering all 4 volumes as potential predictor variables, and NEO agreeableness was used as the single dependent variable.

\section{Results}

The mean NEO agreeableness score for the group was $29.64 \pm 8.00$. The mean volumes for the amygdalae were $1.86 \pm 0.59 \mathrm{~cm}^{3}$ on the right, and $1.56 \pm 0.45 \mathrm{~cm}^{3}$ on the left. Mean medial OFC volumes were $3.57 \pm 0.76 \mathrm{~cm}^{3}$ on the right, and $3.41 \pm 0.79 \mathrm{~cm}^{3}$ on the left. First-order correlations among the 4 brain regions were in the moderate range. Right and left OFC volumes were positively correlated with each other $(\mathrm{r}=0.611, \mathrm{p}<0.001)$, as were right and left amygdalae $(r=0.678 ; p<0.001)$. However, OFC volumes were not significantly correlated with amygdala volumes, regardless of hemispheric laterality.

Linear regression analysis revealed that $38 \%$ of the variance in the NEO agreeableness score was predicted by a model in which right orbitofrontal volume $(\beta=0.731)$ was positively correlated with agreeableness, and left orbitofrontal lobe volume $(\beta=-0.638)$ was negatively correlated with agreeableness $(\mathrm{p}<0.01$; fig. $2 \mathrm{a}, \mathrm{b})$. Contrary to our hypothesis, amygdala volume did not significantly predict agreeableness.

\section{Discussion}

The primary findings from this study were that (1) only OFC volumes were significantly related to NEO agreeableness scores in FTD patients, while amygdala volumes did not show a significant relationship to agreeableness, and (2) the right and left orbitofrontal lobes were oppositely related to the NEO agreeableness score, with smaller right $\mathrm{OFC}$ volume predicting lower agreeableness, and smaller left OFC volume predicting higher agreeableness in FTD patients.

This study partly replicates a previous study that used a different questionnaire of social functioning to delineate a left frontal-right amygdala circuit for warmth/agreeableness in FTD patients [7]. The lack of relationship between agreeableness and the amygdalae may have occurred because the construct of agreeableness measured by the two personality questionnaires is essentially different. The construct measured by the IAS in the pilot study directly implies emotional warmth (e.g. 'tender', 'softhearted') [6], while the NEO-FFI implies cooperative and socially appropriate behavior (e.g., 'I generally try to be thoughtful and considerate'). The current study also showed that the previously documented negative relationship between agreeableness and left frontal lobe volume may actually be restricted specifically to the left OFC. This finding was particularly striking, as the group means for the right and 
left OFC volumes were significantly positively correlated with each other.

Only recently have studies begun to show a divergence between the functions of the right and left orbitofrontal lobes in maintaining social skills. Tranel et al. [15] showed that patients with predominantly left OFC lesions maintained normal social and occupational functioning after their injury, while patients with right OFC damage were unable to maintain their occupational status and evidenced dramatic worsening of social functioning. In the current study, smaller right OFC volumes predicted worse scores on the NEO agreeableness scale, consistent with the findings of Tranel et al. [15] that the right OFC contributes significantly to better interpersonal sensitivity and more effective social functioning. It also supports studies showing the right OFC to be involved in the perspective taking aspects of cognitive empathy [5]. However, our finding that the loss of left OFC volume may correspond to greater levels of agreeableness is novel, and may suggest that the left OFC plays a specific role in self-interested behaviors (awareness of personal needs, assertiveness) that would counterbalance the other-oriented trait of agreeableness. If this is true, the loss of left OFC may cause a functional facilitation of agreeableness, particularly when the right OFC remains intact. Though damage to the left OFC may not significantly impact social functioning compared to the dramatic effects of right-sided damage, the current study suggests that the left OFC plays a more subtle role in personality.

The construct of agreeableness, measured by the NEOFFI, is designed to include the subfacets trust, straightforwardness, altruism, compliance, modesty, and tendermindedness. Thus, the positive relationship between NEO agreeableness and the right OFC suggests that this cortical area mediates cooperative and socially appropriate behavior, particularly behaviors requiring a complex weighing of personal needs and desires against social norms and the needs of others. Patients with lesions to the ventromedial area of the prefrontal cortex have repeatedly been shown to exercise poor judgment in decision making and inappropriate behavior with others [16, 17]. Some have hypothesized that this is due to the role of the medial OFC in a frontal-amygdala-insular circuit that provides feedback about somatic state activation aiding in social decision making [18]. Others have suggested that the OFC may be part of a 'mentalizing circuit', activated upon imagining another person's perspective ('theory of mind') [19], while other theory of mind studies have implicated more dorsal frontal regions, particularly Brodmann's area $8 / 9$ [20-22]. The right OFC is involved in the interpreta- tion of facial emotions in interaction with the amygdala and temporal structures [23], and it has been suggested that OFC activity, in interpreting and imagining the punitive emotional expressions of others, inhibits one from violating social norms [24]. The area measured here included only the most medial two orbitofrontal gyri, suggesting that even changes to this small area can have a significant clinical impact.

The high degree of overlap that has been demonstrated in studies of behavioral features of frontal vs. temporal FTD, such as impaired judgment, sociopathic behavior, loss of social awareness, and social withdrawal $[1,2,25$, 26], may occur because OFC damage is mediating these behaviors in both FTD variants [27]. Also, if the left and right medial OFC are responsible for different social functions, asymmetry of this structure can provide important information in the clinical evaluations of individual FTD patients.

These data support the hypothesis that regulation of agreeableness arises from a balanced, mutually inhibitory circuit involving bilateral cortical structures. This is consistent with numerous recent fMRI discoveries surrounding a variety of higher social functions such as self-reflection, empathy, embarrassment [24], moral reasoning [28], and theory of mind $[21,22]$. Future research using clinical populations to characterize brain-behavior relationships must consider that the neural circuits underlying these higher social functions will not be limited to one brain region, lobe, or even hemisphere. Because of the strong relationship in FTD between altered social behavior and brain atrophy, this population is ideal to further elucidate these complex circuits.

\section{Acknowledgements}

This research was supported in part by the National Institute on Aging (NIA) grants 1 K23 AG21606-01 and AG19724-01A1, the Alzheimer's Disease Research Center of California (ARCC) grant 01154-20, and the Larry L. Hillblom Foundation, Inc., grant 2002/2J. 


\section{References}

1 Miller BL, Chang L, Mena I, Boone K, Lesser IM: Progressive right frontotemporal degeneration: Clinical, neuropsychological and SPECT characteristics. Dementia 1993;4:204-213.

2 Bozeat S, Gregory CA, Ralph MA, Hodges JR: Which neuropsychiatric and behavioural features distinguish frontal and temporal variants of frontotemporal dementia from Alzheimer's disease? J Neurol Neurosurg Psychiatry 2000; 69:178-186.

3 Rankin KP, Kramer JH, Mychack P, Miller BL: Double dissociation of social functioning in frontotemporal dementia. Neurology 2003; 60:266-271.

4 Perry RJ, Rosen HR, Kramer JH, Beer JS Levenson RL, Miller BL: Hemispheric dominance for emotions, empathy and social behaviour: Evidence from right and left handers with frontotemporal dementia. Neurocase 2001;7: 145-160.

5 Rankin KP, Gorno-Tempini ML, Weiner MW, Miller BL: Neuroanatomy of impaired empathy in frontotemporal dementia. 55th Annu Meet Am Acad Neurol, Honolulu, 2003.

6 Wiggins JS: Interpersonal Adjectives Scale: Professional Manual. Odessa, Psychological Assessment Resources, Inc, 1995.

7 Rankin KP, Rosen HJ, Kramer J, Miller B Neuroanatomical substrates of personality change in frontotemporal dementia. Annu Meet Cogn Neurosci Soc, San Francisco, 2002.

8 Costa PTJ, McCrae RR: The NEO Personality Inventory Manual. Odessa, Psychological Assessment Resources, 1985.

9 Brun A, Englund B, Gustafson L, Passant U, Mann DMA, Neary D, et al: Clinical and neuropathological criteria for frontotemporal dementia. J Neurol Neurosurg Psychiatry 1994; 57:416-418.

10 Neary D, Snowden JS, Gustafson L, Passant U, Stuss D, Black S, et al: Frontotemporal lobar degeneration: A consensus on clinical diagnostic criteria. Neurology 1998;51:1546-1554.
11 Costa PT, McCrae RR: Normal personality assessment in clinical practice: The NEO Personality Inventory. Psychol Assess 1992;4:1355 .

12 Schroeder ML, Wormworth JA, Livesley WJ: Dimensions of personality disorder and their relationships to the Big Five Dimensions of personality. Psychol Assess 1992;4:47-53.

13 Ben-Porath YS, Waller NG: 'Normal' personality inventories in clinical assessment: General requirements and the potential for using the NEO personality inventory. Psychological Assessment 1992;4:14-19.

14 Watson C, Andermann F, Gloor P, Jones-Gotman M, Peters T, Evans A, et al: Anatomic basis of amygdaloid and hippocampal volume measurement by magnetic resonance imaging. Neurology 1992;42:1743-1750.

15 Tranel D, Bechara A, Denburg NL: Asymmetric functional roles of right and left ventromedial prefrontal cortices in social conduct, decision-making, and emotional processing. Cortex 2002;38:589-612.

16 Stuss DT, Gow CA, Hetherington CR: 'No longer Gage': Frontal lobe dysfunction and emotional changes. J Consult Clin Psychol 1992;60: 349-359.

17 Blair RJR, Cipolotti L: Impaired social response reversal: A case of 'acquired sociopathy'. Brain 2000;123:1122-1141.

18 Bar-On R, Tranel D, Denberg NL, Bechara A: Exploring the neurological substrate of emotional and social intelligence. Brain 2003;126: 1790-1800.

19 Baron-Cohen S, Ring HA, Moriarty J, Schmitz B, Costa D, Ell P: The brain basis of theory of mind: The role of the orbito-frontal region. $\mathrm{Br} \mathrm{J}$ Psychiatry 1994:165:640-649.

20 Fletcher PC, Happe F, Frith U, Baker SC, Dolan RJ, Frackowiak RS, et al: Other minds in the brain: A functional imaging study of 'theory of mind' in story comprehension. Neuropsychologia 1995;11:141-157.
21 Castelli F, Happe F, Frith U, Frith CD: Movement and mind: A functional imaging study of perception and interpretation of complex intentional movement patterns. Neuroimage 2000;12:314-325

22 Gallagher HL, Happe F, Brunswick N, Fletcher PC, Frith U, Frith CD: Reading the mind in cartoons and stories: An fMRI study of 'theory of mind' in verbal and nonverbal tasks. Neuropsychologia 2000;38:11-21.

23 Iidaka T, Omori M, Murata T, Kosaka H, Yonekura Y, Okada T, et al: Neural interaction of the amygdala with the prefrontal and temporal cortices in the processing of facial expressions as revealed by fMRI. J Cogn Neurosci 2001; 13 : 1035-1047.

24 Berthoz S, Armony JL, Blair RJR, Dolan RJ An fMRI study of intentional and unintentional (embarrassing) violations of social norms. Brain 2002; 125:1696-1708.

25 Miller BL, Cummings JL, Villanueva-Meyer J, Boone K, Mehringer CM, Lesser IM, et al: Frontal lobe degeneration: Clinical, neuropsychological, and SPECT characteristics. Neurology 1991;41:1374-1382.

26 Edwards-Lee T, Miller BL, Benson DF, Cummings JL, Russell GL, Boone K, et al: The temporal variant of frontotemporal dementia. Brain 1997;120:1027-1040.

27 Rosen HJ, Gorno-Tempini ML, Goldman WP Perry RJ, Schuff N, Weiner M, et al: Patterns of brain atrophy in frontotemporal dementia and semantic dementia. Neurology 2002;58:198208.

28 Moll J, Oliveira-Souza R, Bramati IE, Grafman J: Functional networks in emotional moral and nonmoral social judgments. Neuroimage 2002;16:696-703. 